\title{
Dry-Coupled Airborne Ultrasonic Inspection Using Coded Excitation
}

\author{
Dayi Zhang, Robert Watson, Jianlin Cao, Taiyi Zhao, Gordon Dobie, Charles MacLeod, Gareth Pierce \\ Centre for Ultrasonic Engineering \\ University of Strathclyde \\ Glasgow, United Kingdom \\ dayi.zhang@strath.ac.uk
}

\begin{abstract}
Unmanned Aerial Vehicles (UAVs) offer significant potential benefits to the inspection of large-scale facilities due to their ability to access areas where manual inspection is not practical. Ultrasonic inspections typically utilise acoustic couplant, placed between the specimen and transducer surfaces, to eliminate any air gap and enable acoustic energy propagation. Conventional ultrasonic inspection UAVs contain a mechanical system to deliver a small quantity of liquid couplant between the transducer and inspection surface. Such mechanisms increase the system payload, resulting in the reduction of UAV flight endurance and inspection efficiency. Any couplant remaining on the surface may also increase the risk of corrosion. Instead of a liquid couplant layer, dry-coupled ultrasonic transducers utilise a thin layer of rubberised material. However, the acoustic characteristics of the conformable materials typically result in dry-coupled transducers with a lower Signal-to-Noise Ratio (SNR) than liquid-coupled sensors. Coded excitation, a pulse compression technology, improves SNR without sacrificing the measurement acquisition rate, as is the case with signal averaging. This paper explores the potential for application of coded excitation to maintain the SNR aboard a UAV deploying a dry-coupled transducer.
\end{abstract}

Index Terms-Dry-coupled Ultrasound Inspection, Coded Excitation, UAV

\section{INTRODUCTION}

UAVs (Unmanned Aerial Vehicles) are receiving increasing attention for use as remote inspection agents in NDT (NonDestructive Testing), due to affordability, safety, and the ability to access areas where manned inspection is not practical. The current state of the art in the field of UAV based NDT focuses on the visual [1], thermographic [2], and ultrasonic inspections [3]. Previous research demonstrated a contactbased ultrasonic inspection, using a conventional transducer with a small amount of liquid couplant and deployed by an autonomous, multi-rotor UAV. The inspection was conducted by a UAV in an indoor laboratory [4], [5].

However, [4] presented a conventional ultrasonic inspection, using a small quantity of liquid couplant placed between the transducer and the inspection surface to eliminate any air gap and enable acoustic energy propagation. Any couplant remaining on the surface following inspection may increase the risk of corrosion, thus entailing a subsequent cleaning operation. Such a methodology increases the complexity of the inspection process, also reducing UAV flight endurance when carrying a liquid couplant dispenser.
An alternative not incurring such disadvantages within the remote operation context is considered in dry-coupled ultrasonic transducers, such as the $25 \mathrm{~mm}$ diameter wheel probe manufactured by Eddyfi [6]. This utilises a rubberised tire instead of a liquid couplant layer to provide matching between the sample and transducer. As with most dry-coupled ultrasonic transducers, challenges to inspection accuracy associated with lower SNR are encountered due to acoustic attenuation within the conformable layer. A strategy to maintain SNR is necessary for reliable inspection, sufficient to meet industrial standards.

Increasing the excitation voltage applied to the piezoelectric element or averaging across a set of repeated signals are common approaches. However, high voltage excitation is undesirable for safe operation within certain explosible industrial environments [7]. Additionally, intrinsic limits to the ability of a conventional multirotor platform to hold position amid aerodynamic disturbances make extensive signal averaging impractical for airborne ultrasonic inspections.

Coded excitation is a more elegant solution to increase SNR within a UAV inspection system. It has been demonstrated within medical imaging [8] and NDT applications [9]. Coded excitation consists of a series of binary bits, each encoding a state of \pm 1 . Multiple pulses are transmitted in series whereby the polarity of the excitation voltage used for each pulse is varied according to the bits of the excitation sequence.

Coded excitation modes, such as the Golay encoding, improve the received SNR without increasing the amplitude of the excitation voltage or averaging across multiple signals and are commonly used in ultrasonic applications [10]. Golay code excitation contains a pair of sequences, coded with two different polarity patterns. Each pattern consists of a length of $L=2^{n}$ bits, where $\mathrm{n}$ is a positive integer. The two complementary signals received after the transmission of these coded sequences thus contain different wave patterns. Decoding the received signals via correlation with their excitation waveform enhances signal content present due to sample boundary reflections but introduces sidelobe distortions. However, the sidelobes of the complimentary signals in a Golay pair are opposite in polarity and so are removed by summation, yielding the final signal. As described by the Golay code definitions [11], the amplitude of the final result at 
zero lag has a constant amplitude of $2 L$. Note that the sequence length $L$ is constrained by the signal processing speed and the transceiver memory space. Fig. 1 presents the 8-bit Golay coded sequence, the autocorrelated signals and the resultant signal after summation.
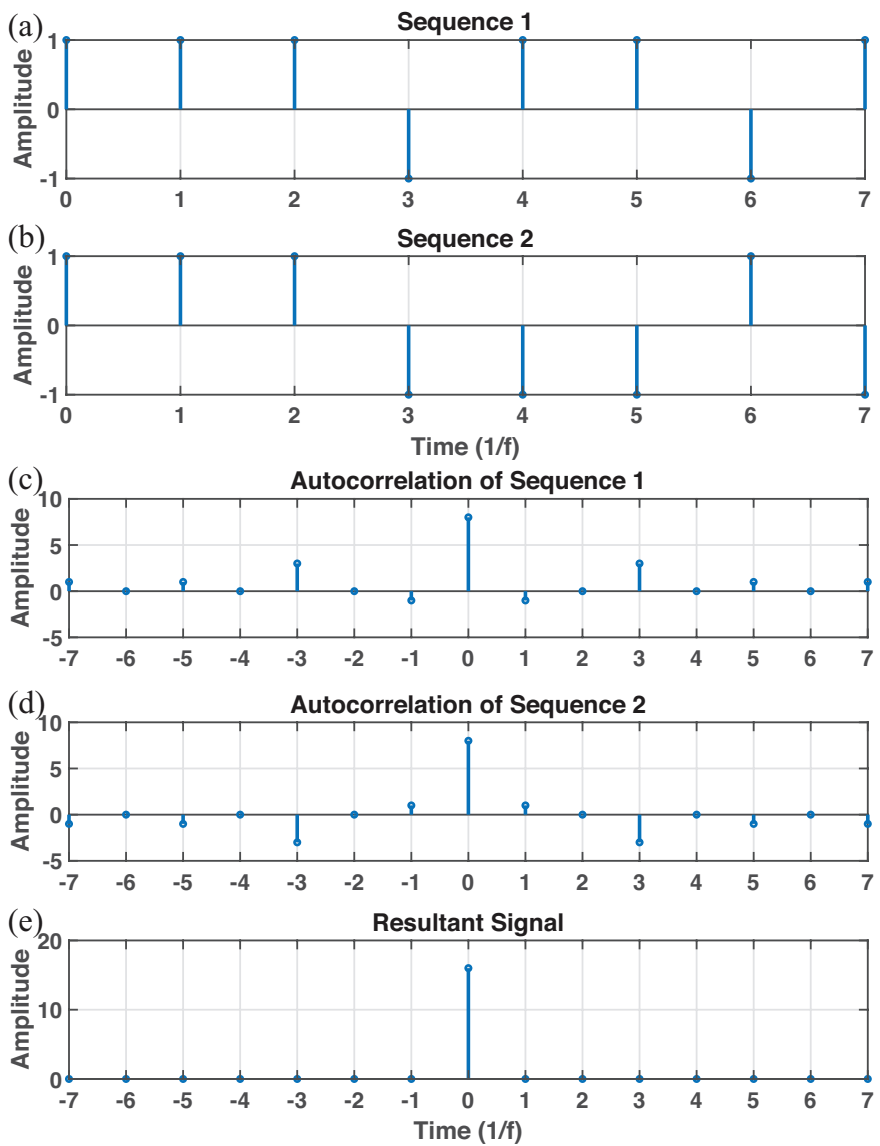

Fig. 1. A pair of 8-bit Golay sequences, (a) (b), autocorrlated signals (c), (d) and the resultant signal (e), where the $\mathrm{f}$ is the transducer centre frequency.

Through this method, the signal-to-noise ratio of the Golay code is increased by a factor of $\sqrt{2 L}$ versus a single excitation. This effect is readily apparent when comparing the noise levels in the amplitude normalised A-scans (maximum amplitude is 1) of a single pulse excitation and the processed Golay sequence generated using consistent excitation voltages, shown in Fig. 2.

This paper evaluates the use of 8-bit Golay sequences using a dry-coupled ultrasound transducer under a contact force provided by a robotic manipulator, which simulates the UAV contact inspections. From signal processing theory, an SNR improvement of $12 \mathrm{~dB}$ is expected under this sequence, matching the improvement found in averaging across 16 repeated signals in $12.5 \%$ of the acquisition time.

\section{Methodology}

Ultrasonic thickness measurement is conducted utilising a dual-crystal, $5 \mathrm{MHz}, 25 \mathrm{~mm}$ diameter, Eddyfi wheel probe [6], driven by a custom transceiver. The transceiver stimulates
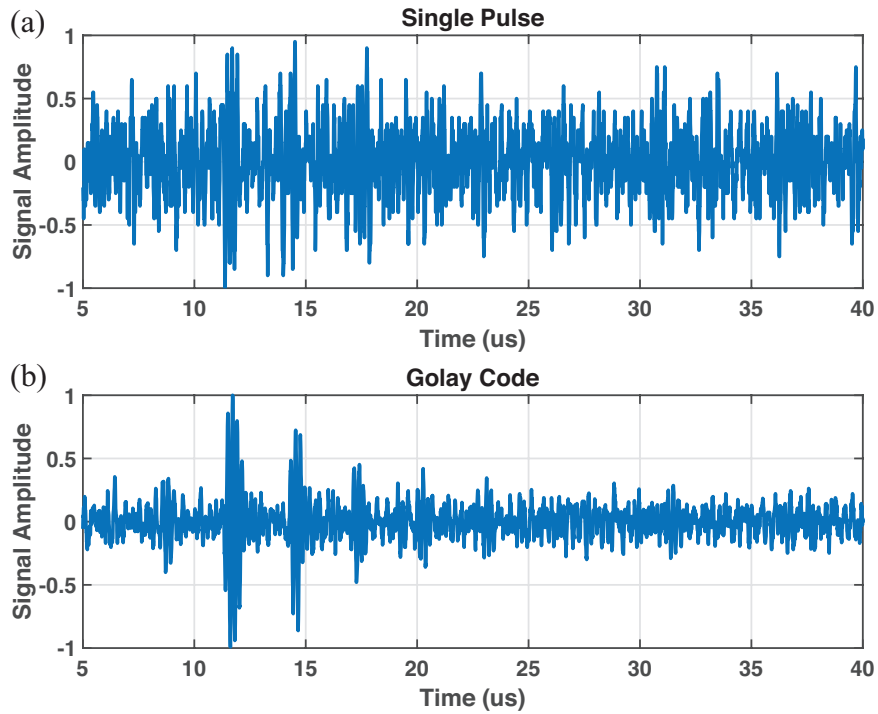

Fig. 2. A-scan signals of (a) single pulse excitation and (b) 8-bit Golay coded excitation.

the dedicated transmission element inside the probe with \pm 12 $\mathrm{V}$ bipolar rectangular waveforms. Bespoke interface software triggers the transducer at a $60 \mathrm{~Hz}$ pulse repetition frequency, transfers the digitised waveform from the transceiver to a computer and performs real-time signal processing to decode the Golay sequences.

A $25 \mathrm{~mm}$ steel plate sample was used as an analogue to an in-situ industrial inspection. The transducer was mounted on the end of a KUKA KR6 R900: an industrial, six-degree of freedom, robotic manipulator arm [12]. This robotic manipulator was used to repeatably deploy the wheel probe to the steel sample at a consistent location and orientation, while applying variable force, deforming the probe tire and acoustically coupling to the sample. As the contact force produced by a conventional multirotor UAV during airborne inspection is relatively low [4], the effects of increasing force in regular increments over a range of 0 to $20 \mathrm{~N}$ are examined. Applied force is measured using a force-torque sensor [13] between the probe and the robot manipulator. The experiment setup is depicted in Fig. 3.

Variation in SNR with contact force is thereby investigated. Here measured SNR is evaluated by dividing the peak amplitude of the reflected signal by the noise amplitude and expressing the result in decibels. The noise amplitude is taken as the maximum absolute value of the received signal in the time before the first received echo.

\section{EXPERIMENT RESULTS}

Using the method described above, contact force between the wheel probe and sample was varied and the ultrasonic signals recorded as tire deformation altered the level of acoustic coupling. Starting with a contact force of $0 \mathrm{~N}$, at the point immediately before the probe began to contact the sample, force was increased through the range of interest, noting the 


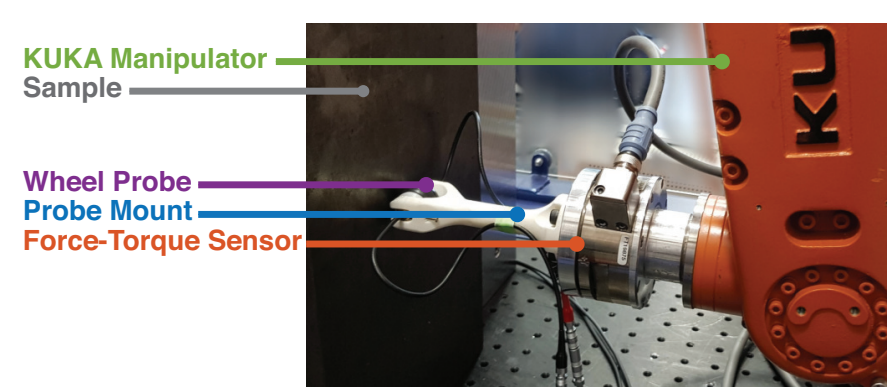

Fig. 3. A photograph of the hardware used in the experiment process.

point at which the instrumentation recorded identifiable backwall echoes in the 8-bit Golay code excited A-scan signal. The minimum force for a meaningful thickness measurement is thus found to be circa $4 \mathrm{~N}$, measured by the force-torque sensor. Fig. 4 depicts this relationship between the contact force and SNR.

In comparison to a single pulse excitation, the SNR is improved by approximately $12 \mathrm{~dB}$ when using the 8-bit Golay code or averaging across 16 single excitations. Note that no distinct echo signal can be recognised at probe contact forces below $4 \mathrm{~N}$, so SNR data from $0 \mathrm{~N}$ to $3 \mathrm{~N}$ are omitted.

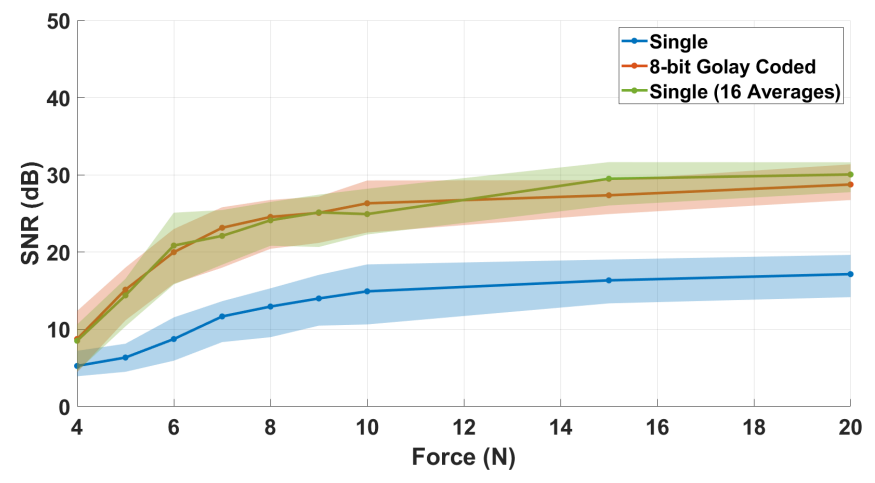

Fig. 4. The relationship between the SNR and measured contact force.

From Fig. 4, it may be observed that the SNR increases rapidly with compressive force between $4 \mathrm{~N}$ and $10 \mathrm{~N}$. In this region, SNR is most strongly influenced by the acoustic transmittance of the dry coupling tyre. A small increase in applied force at this level causes a significant increase in the tyre deformation, and hence its area in contact with the sample surface, permitting transmission of a greater fraction of the ultrasonic signal energy. At forces above $10 \mathrm{~N}$, the probe is fully coupled to the sample and additional pressure offers diminished SNR increase. These results show that the coded excitation strategy offers improved SNR performance across the entire range of coupling forces that may be generated by a conventional multirotor UAV deploying a dry coupled probe.

Fig. 5 presents example signals captured in selected combinations of excitation mode and contact force for visual comparison. Under the 8-bit Golay excitation with $4 \mathrm{~N}$ force of Fig. 5(b) the SNR was $11.03 \mathrm{~dB}$, greatly increased from 4.74 $\mathrm{dB}$ as captured using a single excitation with the same force,
Fig. 5(a). Additionally, the thickness cannot be accurately inferred from the single pulse excitation of Fig. 5(a) due to a lack of identifiable repeat back-wall echoes. The SNR of the Golay-coded excitation is comparable to the results presented in [4], where the inspection was deployed by a GB Inspection manufactured [14], $5 \mathrm{MHz}, 10 \mathrm{~mm}$-diameter, conventional dual-crystal transducer using liquid coupling.

Increasing the contact force to $8 \mathrm{~N}$, the single pulse excitation, Fig. 5(c), achieved SNR of $11.82 \mathrm{~dB}$ : a similar result to the Golay coded excitation at $4 \mathrm{~N}$. Under this contact force, the SNR of 8-bit Golay code was increased by $12.83 \mathrm{~dB}$ to 24.65 $\mathrm{dB}$. More back-wall echoes were visible to the transceiver allowing for accurate thickness measurement in support of NDT activities.
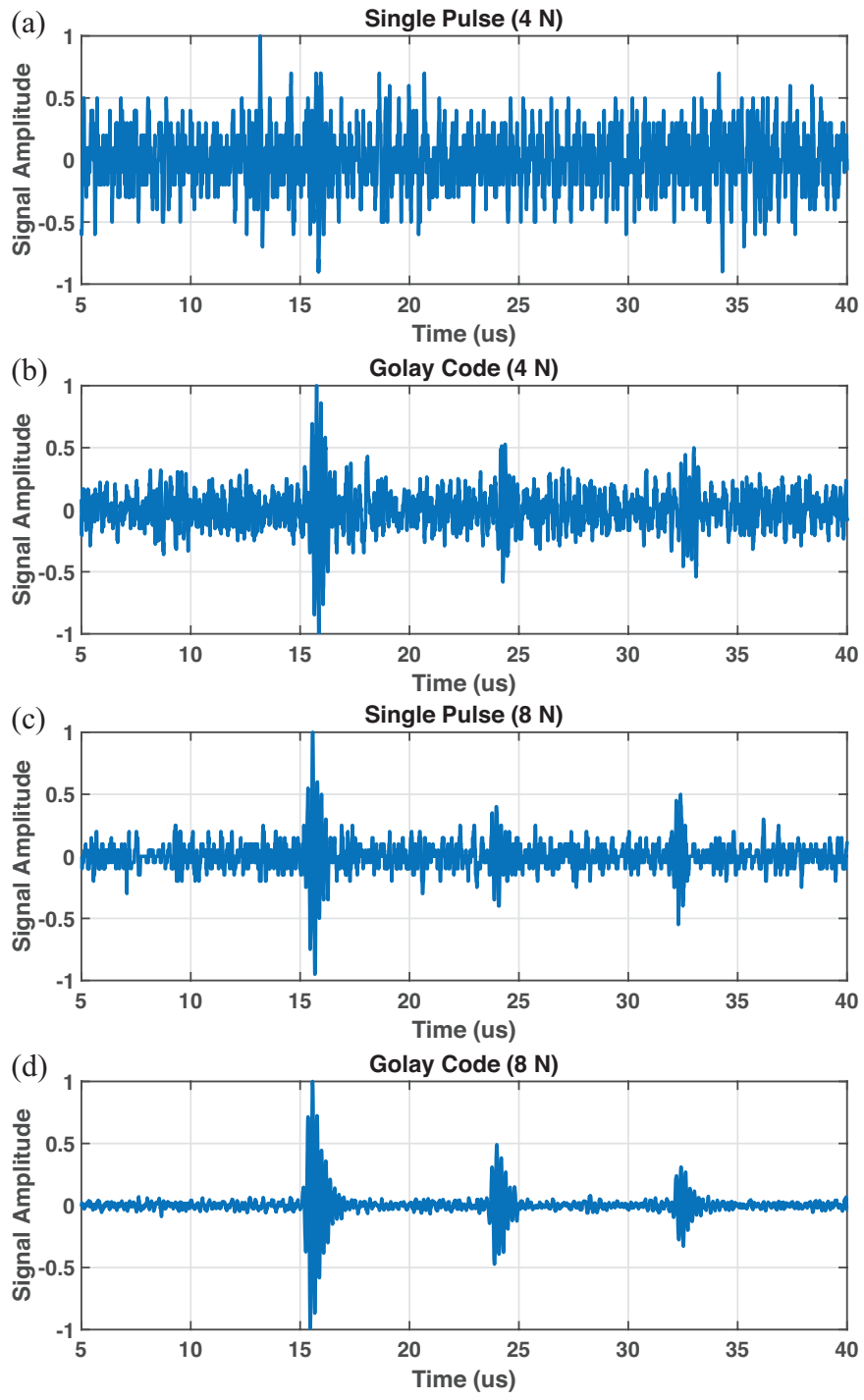

Fig. 5. A-scan signals of (a) single pulse excitation with $4 \mathrm{~N}$ force applied and (b) 8-bit Golay coded excitation with $4 \mathrm{~N}$ force applied (c) single pulse excitation with $8 \mathrm{~N}$ force applied and (d) 8-bit Golay coded excitation with $8 \mathrm{~N}$ force applied 


\section{Discussion AND CONCLUSIONS}

The coded excitation system was successfully deployed using a dry-coupled ultrasonic wheel probe, achieving a similar SNR to a previously obtained result using a conventional liquid coupled ultrasound transducer [4]. With the $24 \mathrm{~V}$ peak-to-peak coded excitation, it was possible to detect ultrasonic echoes at a contact force of only $4 \mathrm{~N}$. This is a significant improvement compared to the $8 \mathrm{~N}$ required by a single pulse excitation of the same instrumentation. Such an approach reduces the contact force required to capture meaningful ultrasonic signals, and so imposes less stringent design requirements on a UAV system for airborne ultrasonic inspection. Within the range of the relatively small contact forces generated by conventional multirotor UAVs, Golay coded excitation is therefore demonstrated as a valid strategy to improve SNR when conducting contactbased ultrasonic inspections. In combination with dry-coupled probes, coded excitation modes offer enhanced potential for airborne ultrasonic inspections without liquid couplant and its required deposition and clean-up processes. This constitutes an opportunity to further improve upon the efficiency of many current industrial operations and maintenance strategies.

\section{REFERENCES}

[1] D. Zhang, R. Watson, G. Dobie, C. MacLeod, A. Khan, and G. Pierce, "Quantifying impacts on remote photogrammetric inspection using unmanned aerial vehicles," Eng. Struct., vol. 209, p. 109940, Apr. 2020.

[2] X. Li, Q. Yang, Z. Chen, X. Luo, and W. Yan, "Visible defects detection based on UAV-based inspection in large-scale photovoltaic systems," IET Renew. Power Gener., vol. 11, no. 10, pp. 1234-1244, 2017.

[3] L. M. González-deSantos, J. Martínez-Sánchez, H. González-Jorge, and P. Arias, "Active UAV payload based on horizontal propellers for contact inspections tasks," Meas. J. Int. Meas. Confed., vol. 165, 2020.

[4] D. Zhang, R. Watson, G. Dobie, C. MacLeod, and G. Pierce, "Autonomous Ultrasonic Inspection Using Unmanned Aerial Vehicle," IEEE Int. Ultrason. Symp. IUS, vol. 2018-Octob, 2018.

[5] D. Zhang et al., "Evaluation of Coded Excitations for Autonomous Airborne Ultrasonic Inspection,” in IEEE International Ultrasonics Symposium, IUS, 2019, vol. 2019-Octob, pp. 2461-2464.

[6] Eddyfi, "Swift and Scorpion2 Remote-Access Tank Shell Inspection Solution," 2018. [Online]. Available: https://eddyfi.com/en/product/scorpion-2/. [Accessed: 01-Aug-2020].

[7] S. Exective, "Electricity at work Safe working practices," 2013. [Online]. Available: http://www.hse.gov.uk/pUbns/priced/hsg85.pdf.

[8] M. O'Donnell, "Coded Excitation System for Improving the Penetration of Real-Time Phased-Array Imaging Systems," IEEE Trans. Ultrason. Ferroelectr. Freq. Control, vol. 39, no. 3, pp. 341-351, 1992.

[9] R. Su et al., "Coded excitation for air-coupled ultrasound phased arrays," 56th Annu. Conf. Br. Inst. Non-Destructive Testing, NDT 2017, 2017.

[10] I. A. Veres et al., "Golay code modulation in low-power laserultrasound," Ultrasonics, vol. 53, no. 1, pp. 122-129, 2013.

[11] B. Zhou, D. M. Green, and J. C. Middlebrooks, "Characterization of external ear impulse responses using Golay codes.," J. Acoust. Soc. Am., vol. 92, no. 2, pp. 1169-71, 1992.

[12] KR AGILUS, "KUKA," 2015. [Online]. Available: https://www.kuka.com/en-de/products/robot-systems/industrialrobots/kr-agilus. [Accessed: 01-Aug-2020].

[13] ATI Industrial Automation, "F/T Sensor: Gamma," 2020. [Online]. Available: https://www.atiia.com/products/ft/ft_models.aspx?id=Gamma. [Accessed: 01-Aug2020].

[14] GB Inspection Systems Ltd., "Compression Wave 0o Probes," 2018. [Online]. Available: https://www.gbinspection.com/products/probesaccessories/compression-wave-0-probes. [Accessed: 01-Aug-2020]. 